\title{
Release notice
}

\section{Data release for the Canadian Longitudinal Study on Aging}

The first major data release from the Canadian Longitudinal Study on Aging (CLSA) is underway. The June 2014 release includes data collected from 21242 participants who each completed a 60-minute telephone interview. Additional data from these interviews will become available early in 2015 .

The process for accessing biospecimens and physical assessment data from an additional 30000 participants who were interviewed in person and have visited one of 11 data collection sites across the country, is currently being developed in anticipation of the first release of these data in 2016 .

Canadian and international public sector researchers interested in accessing the CLSA platform are invited to visit the DataPreview Portal on the CLSA website for detailed information about the available data and the application process.

Data will be available to researchers following review of applications by the CLSA Data and Sample Access Committee. For more information, visit www.clsa-elcv.ca. 